\title{
UNE ANALYSE PRAGMATIQUE DE LA PRESSE BOURSIÈRE
}

\author{
Aude Seron et Piotr Jan Karp ${ }^{1}$
}

\section{Un ratissage en quatre temps}

Dans le ratissage pragmatique du corpus d'articles concerné ${ }^{2}$, nous avons procédé, comme son nom l'indique, à l'étude du positionnement des interlocuteurs de la presse boursière et cela en vue de déterminer la modalité relationnelle instaurée par le discours de cette presse entre le journaliste et le lecteur. Reprenant les deux grandes catégories établies au moment de la composition du corpus entre «la presse boursière spécialisée » et « la presse boursière non spécialisée $»^{3}$, nous avons circonscrit notre analyse textuelle à quatre aspects énonciatifs signifiants : les pronoms personnels, les intervenants, les marques journalistiques et les actes du discours. En finale, cet angle d'approche nous a permis d'établir certaines corrélations entre le positionnement des interlocuteurs et les opportunités d'apprentissages informels offertes au lectorat. Comme le souligne très justement Dominique Maingueneau : « À chacun des statuts [assignés aux interlocuteurs] sont attachés des droits et devoirs, mais aussi des savoirs ${ }^{4}$.»

1 Aude SERON, assistante de recherche au Département de communication de l'UCL ; Piotr Jan KARP, étudiant du DEA au Département de communication de l'UCL.

2 Nous renvoyons nos lecteurs à la présentation détaillée du corpus d'articles boursiers qui figure en introduction des contributions étudiantes.

3 Cette double dimension présentée dans la partie descriptive du corpus rejoint également les classifications habituelles, et notamment celles proposées par S. GUERIN, La presse économique et financière, Paris, Editions du Centre de formation et de perfectionnement des journalistes, 1991.

4 D. MAINGUENEAU, Analyser les textes de communication, Paris, Editions Dunod,

Recherches en communication, $\mathrm{n}^{\circ} 23$ (2005). 


\section{Les pronoms personnels}

Cette première dimension de l'analyse se fonde sur l'idée selon laquelle l'ensemble des énoncés émanent d'un destinateur et s'adressent à un destinataire, ces interlocuteurs apparaissant de façon manifeste dans les pronoms personnels qui les représentent. Les travaux de divers auteurs, dont ceux du linguiste Emile Benveniste ${ }^{1}$ pour ne citer qu'un grand nom, ont permis de corréler à un pronom personnel bien spécifique, un mode de relation ou de positionnement précis. Rappelons brièvement que la première personne du singulier, le pronom «je », introduit une centration sur le destinateur qui se révèle explicitement par cet usage et s'implique fortement dans le discours. Dans sa forme plurielle, actualisée par le pronom «nous », le « je » s'annexe d'autorité une communauté qui lui est distincte, le destinateur perpétuant ainsi - mais de façon moins marquée - la centration sur lui : « d'une manière générale, la personne verbale au pluriel exprime une personne amplifiée et diffuse ; c'est un je dilaté au-delà de la personne stricte, à la fois accru et de contours vagues ${ }^{2}$. » L'annexion communautaire caractéristique de la première personne du pluriel se déploie à la fois sous une forme inclusive équivalant au «moi+vous » et une forme exclusive assimilable au « moi+eux ${ }^{3} »$. L'emploi de la seconde personne, le pronom « tu », conserve la même force mais appliquée cette fois au destinataire par la volonté du destinateur. Il en résulte donc une émergence tacite de l'interlocuteur ainsi que le désir du locuteur d'impliquer fortement celui-ci dans son propos. Des constats similaires s'appliquent à la forme plurielle que le pronom « vous » concrétise tant dans son acception singulière de politesse que dans sa visée collective. Contrairement aux deux premières personnes du singulier, « je » et « tu », la troisième incarnée par le pronom «il », ne désigne pas un individu particulier. De l'élévation révérencieuse traduisant un usage poli, à l'exclusion méprisante de la sphère du discours, l'interlocuteur ainsi actualisé se

1998, p. 52.

1 E. BENVENISTE, Problèmes de linguistique générale, vol.1, Paris, Editions Gallimard, 1973 ; vol. II, Paris, Editions Gallimard, 1974.

2 E. BENVENISTE, Problèmes de linguistique générale, Paris, Editions Gallimard, 1996, p. 234-235, cité par D. MAINGUENEAU, op.cit., p. 105.

3 J-P., MEUNIER, D. PERAYA, Introduction aux théories de la communication, : analyse sémio-pragamtique de la communication médiatique, Bruxelles, De Boeck, 1993, p. 72. 
voit qualifié de manière impersonnelle. Enfin, le pronom « ils » « (...) désigne une collectivité, une pluralité prise globalement et constituée d'individus indéterminés ${ }^{1}$, ce qui revient à dire que la modalité plurielle de la troisième personne constitue une extension plus générale du pronom « on ». En raison de sa dépersonnalisation, le « on » possède la faculté de se substituer à l'ensemble des pronoms que nous venons de passer en revue.

Partant des divers degrés d'implication relationnelle qui distinguent ces pronoms, nous avons isolé parmi ceux-ci un sous-ensemble de trois pronoms considérés comme les plus impliquants -en termes de positionnement des interlocuteurs- et dont le crescendo s'établit du « on » vers le « vous» en passant par le «nous». Nos analyses nous ont permis de tirer un ensemble de constats différenciés entre les articles répertoriés dans la presse spécialisée et ceux rattachés à la catégorie de la presse non spécialisée.

\section{La presse spécialisée}

S'agissant de la presse spécialisée (Trends Tendances, L'Echo et La Libre Entreprise), nous avons noté une présence globalement faible des trois pronoms impliquants visés. Cette apparente homogénéité se nuance cependant légèrement selon les journaux. On observe de fait une croissance régulière de ce mode d'implication personnelle en partant des articles du Trends Tendances vers ceux de L'Echo, en passant par ceux de La Libre Entreprise ${ }^{2}$.

Si l'on précise les modalités selon lesquelles cette faible présence se répartit en termes de catégories pronominales, on constate ceci. D'une part et tous articles confondus, on note l'absence totale du pronom « vous » le plus impliquant, à l'exception d'un intertitre dans un article du Trends Tendances:

«Otez donc toutes ces couches!»

(Trends Tendances, «La révolte des actionnaires », 24/05/2001)

Dans la mesure où l'intertitre constitue un espace propice à l'accroche, il ne possède pas la même force d'implication qu'une phrase insérée au sein même de l'article et mérite à ce titre d'être relativisé.

D. MAINGUENEAU, op.cit., p. 113.

2 On note en moyenne de 1 à 5 pronoms impliquants par article dans Trends Tendance, de 1 à 8 pronoms du même type dans L'Echo et de 3 à 11 dans La Libre Entreprise. 
D'autre part, on observe la présence récurrente mais néanmoins faible du pronom «nous ${ }^{1}$. Enfin, on constate la présence récurrente et plus ou moins importante du pronom « on » le moins personnellement impliquant ${ }^{2}$.

On voit donc émerger une timide présence journalistique à ce niveau pronominal et tendant, lorsqu'elle se marque le plus, à privilégier la forme d'implication relationnelle la moins forte, à savoir le « on ». Si l'on se réfère à Dominique Maingueneau, ce pronom permet au locuteur de s'adresser à un individu (une subjectivité) sans pour autant lui accorder le statut d'un véritable co-énonciateur. L'usage du pronom «on» permet au locuteur «de se situer sur une frontière $»^{3}$ entre point de vue extérieur et point de vue de l'interlocuteur : tout en se plaçant au point de vue de son interlocuteur, le locuteur conserve sa propre position.

\section{La presse non spécialisée}

Contrairement aux articles de la presse spécialisée, les articles issus de la presse non spécialisée délaissent l'homogénéité pronominale au profit de deux logiques relationnelles distinctes.

La première logique caractérise les articles de La libre Belgique et se traduit par une absence presque uniforme des pronoms «on», « nous » et « vous »: un seul « on» est répertorié sur les trois articles tirés de ce quotidien. Cette tendance définit un énonciateur -et plus largement des interlocuteurs- particulièrement effacé. La seconde logique concerne les articles du Vif/L'Express qui accueillent quant à eux un nombre significatif de pronoms impliquants ${ }^{4}$ en multipliant en outre l'usage du pronom « vous » ${ }^{5}$. Le journaliste énonciateur interpelle donc ici de façon particulièrement directe son lecteur :

« Alors accrochez vos ceintures et envolez-vous pour un monde technologique. Mais gare aux trous d'air. Ils semblent de plus en plus inévitables. »

(Le Vif/L'Express, «Le Nasdaq contre le Dow Jones », 03/03/2000)

\footnotetext{
En moyenne de 1 à 3 pronoms par article

En moyenne de 1 à 9 pronoms par article.

D. MAINGUENEAU, op.cit., p. 112.

De 4 à 21 pronoms par article.

De 1 à 14 pronoms par article.
} 


\section{Les intervenants}

Ce deuxième niveau d'analyse concerne l'ensemble des protagonistes appelés à intervenir dans le cadre de l' article. Plus concrètement, il s'agit des « sources » auxquelles le journaliste se réfère pour étayer son propos. Les intervenants regroupent donc généralement des spécialistes de la question abordée : des acteurs d'une entreprise ou d'une institution, des professeurs, des chercheurs, des analystes, des consultants, etc., impliqués dans le domaine abordé. Nos analyses nous ont permis de caractériser les intervenants au départ de cinq grands critères : leur nombre, la forme de leur discours, leur degré de présence, le dévoilement de leur identité et leur statut. L'aspect numérique renvoie à l'unicité ou à la multiplicité des intervenants sollicités au sein d'un article. La forme du discours se rattache à la distinction entre le discours rapporté direct et le discours rapporté indirect. Le degré de présence d'un intervenant s'évalue à l'abondance référentielle que le journaliste manifeste à son égard. La dimension identitaire traduit la précision avec laquelle l'intervenant se trouve nommé et précisément présenté -du point de vue sa fonction professionnelle notamment- par le journaliste. Enfin, le statut apparaît comme un corollaire de l'identité, référant au prestige, à la reconnaissance sociale ou institutionnelle dont bénéficie l'intervenant et dès lors aussi au crédit que le lecteur peut accorder aux dires de celui-ci.

\section{La presse spécialisée}

Au niveau de la presse spécialisée on observe un recours systématique aux intervenants et plus précisément encore aux intervenants prestigieux qui englobent les intervenants personnellement nommés, occupant une fonction clairement définie et importante.

« Roberto Colaninno, patron de Telecom Italia, a clairement exprimé sa colère à l'égard de Liverpool Partners, un hedge fund basé aux Bermudes... » (Trends Tendances, La révolte des actionnaires, 24 mai 2001)

«Par conséquent, les niveaux de valorisation actuels sont attractifs », précisent, dans une note de recherche, Caroline Lauwers et Jean-Etienne Pierre, analystes auprès de KBC Securities » (L'Echo, «Belle croissance bénéficiaire en perspective pour les distributeurs alimentaires européens », 07/06/2001) 
À l'exception des articles de L'Echo qui recourent unanimement à un ou deux intervenants privilégiés et qui suivent de très près leurs $\operatorname{propos}^{1}$, les autres journaux laissent apparaître toutes sortes de variations d'un article à l'autre ne permettant pas véritablement de dégager une tendance prédominante.

La place de choix laissée aux intervenants prestigieux constitue selon nous un indicateur significatif de l'importance globalement accordée par la presse spécialisée à la logique d'authentification des propos journalistiques.

\section{La presse non spécialisée}

Deux modes de fonctionnement différents ressortent de l'analyse des articles de la presse non spécialisée. D'un côté, les articles de la Libre Belgique s'inscrivent dans la lignée de la presse spécialisée en recourant le plus souvent à des intervenants. Mais ce recours n'est plus systématique et par ailleurs, il s'agit autant d'intervenants prestigieux et clairement définis que d'intervenants se rattachant à une catégorie vague d'acteurs tels que « les analystes » par exemple. De l'autre côté, les articles du Vif/L'Express se démarquent de la tendance générale en délaissant les intervenants qui font figure d'exception dans ce sousgroupe de presse $^{2}$ et demeurent tous peu définis : leur caractérisation se résume par une appellation renvoyant au nom d'une institution bancaire ou à une catégorie générique d'acteurs comme «les analystes », «les habitués de la Bourse », etc. En outre, les rares intervenants qui émergent dans le Vif ancrent plutôt des adages renvoyant à une forme de sagesse populaire :

«( ...) il faut (...) appliquer l'adage bien connu des habitués de la Bourse : «Acheter au son du canon et vendre au son du clairon » ». (Le Vif/L'Express, «Acheter au son du canon ? », 26/01/2001)

« «Don't fight the Fed», commentent les spécialistes : ne combattez pas la politique de la banque centrale. »

(Le Vif/L'Express, «Acheter au son du canon ? », 26/01/2001)

1 Ce constat repose très vraisemblablement sur une justification contextuelle dans la mesure où tous ces articles présentent la particularité de relater les résultats d'une étude ou d'une enquête réalisée par une institution liée au milieu économique et financier.

2 On note à peine la présence de 3 intervenants sur l'ensemble des 5 articles. 
Par ce biais on peut estimer que si les articles du Vif s'inscrivent d'une certaine manière aussi dans une logique d'authentification des propos journalistiques, cette dernière se fonde davantage sur le «bon sens » que sur le prestige des intervenants.

\section{Les marques journalistiques}

Sous l'appellation de marques journalistiques nous avons procédé au regroupement subjectif de différents éléments.

D'une part, figurent dans cet ensemble toute une série de marques de ponctuation possédant pour l'essentiel soit une visée authentifiante (lorsqu'elles visent à légitimer le discours journalistique), soit une visée inférentielle (lorsque ces marques cherchent plutôt à susciter dans le chef du lecteur un travail réflexif qui reste le plus souvent déductif). S'agissant de la visée inférentielle, elle renvoie à l'idée soutenue par Dominique Maingueneau ${ }^{1}$ selon laquelle en attirant l'attention du lecteur, l'énonciateur veut signifier à ce dernier qu'il existe là un manque qu'il se doit de combler par lui-même. Concrètement, ce premier sousensemble de marques concerne les parenthèses, les tirets, les guillemets, la police des caractères (italiques, gras, majuscule, etc.) les points de suspension et enfin les exclamations. Le passage suivant illustre une combinaison d'exclamations et de mises entre parenthèses révélant non seulement le positionnement du journaliste mais aussi un complément d'information visant à induire un effet implicite d'étonnement, de sidération chez le lecteur :

«Par la seule présence d'Interbrew et de ce mécanisme juridique, la présence néerlandaise à Bruxelles est plus que triplée à 9,7\% (contre $3 \%$ en 1999) ! »

( L'Echo, «La présence d'Interbrew chamboule les statistiques d'Euronext Bruxelles », 30/05/2001)

Parmi, toutes ces marques, nous nous sommes attachés à ne traiter que les éléments les plus impliquants au niveau relationnel, c'est-à-dire ceux qui traduisaient dans le contexte de l'article l'une ou l'autre des deux visées identifiées.

D'autre part, nous avons inclus dans la catégorie des marques journalistiques les formulations interrogatives qui se rattachent également à

1 D. MAINGUENEAU, op. cit, p. 138. 
une forme particulière d'actes du discours et qui constituent une marque fortement impliquante.

Le troisième sous-groupe constitutif des marques journalistiques englobe les références, c'est-à-dire les différents types de renvois à une source extra-textuelle quelconque. Cela peut s'illustrer par l'extrait suivant :

«Inutile de dire que le coût relatif de ces clients pour les sites lancés récemment comme 12trade.be (groupe ING) dont le dernier relevé (source : « De Tijd », décembre 2000) n'est fort que de 2.600 clients. »

(La Libre Entreprise, « Brokers online en panne », 10/03/2001)

Enfin, nous avons également pris en compte la signature des articles en distinguant le degré de précision de celle-ci et le nombre d'auteurs impliqués : initiales personnelles, groupe de presse, signature journalistique individuelle ou collective.

\section{La presse spécialisée}

Une fois n'est pas coutume, la presse spécialisée se départit ici de sa tendance à l'homogénéité au profit d'une double expression relationnelle.

Alors que les articles du Trends Tendances se caractérisent par une présence particulièrement faible des marques journalistiques toutes sous-catégories confondues ${ }^{1}$ on note par contre une présence relativement importante de ces marques dans les articles de $L^{\prime} E c h o^{2}$ ainsi que dans ceux de La Libre Entreprise ${ }^{3}$.Toutefois, le consensus qui lie à ce niveau L'Echo et La Libre Entreprise ne porte que sur l'aspect numérique des marques journalistiques. Si L'Echo privilégie les marques d'authentification du discours journalistique ${ }^{4}$ sans trop laisser transparaître le positionnement du journaliste, y compris au niveau de la signature qui identifie peu souvent le journaliste de façon précise $^{5}$, La Libre

\footnotetext{
On note de 3 à 6 marques par article.

On compte de 3 à 22 marques par article.

On observe de 14 à 27 marques par article.

Notamment les références et les mises en évidence par le biais des guillemets et des parenthèses.

5 La signature n'apparaît de façon complète qu'une fois sur trois.
} 
Entreprise multiplie quant à elle les marques les plus impliquantes ${ }^{1}$ et laisse plus clairement émerger le positionnement (l'avis) du journaliste sur le sujet traité, y compris au niveau de la signature dans laquelle le journaliste apparaît chaque fois de façon nominale. Ce positionnement journalistique engagé transparaît de façon assez claire dans l'exemple suivant :

«Quitréédpointcom, ça sonne bien en radio, mais pour retrouver le site... (...) Bravo par contre pour la dmrj.com de la société de Bourse De Moffart, Rolin Jacquemyns : si dmrj n'a pas de sens pour le surfeur-investisseur, l'adresse est transparente. »

(La Libre Entreprise, « Brokers online en panne », 10/03/2001)

Tous ces constats nous incitent donc plutôt à conclure à un usage singulier des marques journalistiques pour chacun des journaux étudiés.

\section{La presse non spécialisée}

Concernant la presse non spécialisée, on se trouve une fois encore face à deux logiques très différentes. D'un côté, La Libre Belgique se distingue par une présence relative mais atténuée des marques journalistiques $^{2}$ y compris au niveau de la signature qui se résume toujours à des initiales. Et de l'autre côté Le Vif/L'Express se différencie par le privilège qu'il accorde aux marques relationnelles fortement impliquantes que sont les questions ${ }^{3}$ et la faible présence des marques authentifiantes comme les références qui deviennent quasiment inexistantes. L'exemple suivant offre une bonne illustration de cette forte implication relationnelle prônée par le style du Vif:

1 Entre autres les interrogations et les suspensions.

2 On note respectivement pour les trois articles de cet hebdomadaire et toutes marques confondues les fréquences d'apparition suivantes : 4, 22 et 4. D'autre part, même si ces marques semblent parfois nombreuses, il semblerait qu'elles visent avant tout à attirer l'attention du lecteur et éventuellement à apporter une emphase au discours. C'est notamment le cas de valeurs chiffrées indiquées en gras (l'un des articles comptabilise jusqu'à 8 de ces marques).

3 On constate dans le détail pour chaque article : 0, 9, 1, 7 et 11 rien que pour les questions et 17, 21, 13, 22, et 29 toutes marques confondues. 
« Reste une difficulté : comment déterminer le contenu et la liste des secteurs? À la différence d'un pays, le concept est moins net, et certaines actions peuvent avoir un profil flou. $\mathrm{Si}$ UCB est davantage une valeur pharmaceutique que chimique, qu'en est-il de Solvay où la pharmacie prend de plus en plus d'importance dans le résultat final ? Cela dit, la classification par pays rencontre les mêmes problèmes. Cas typique en Belgique ?»

(Le Vif/L'Express, « Une stratégie payante », 23/04/1999)

\section{Les actes du discours}

Dans ce quatrième volet analytique, nous nous sommes uniquement intéressés à la catégorie des actes illocutoires ou illocutionnaires et cela dans la mesure où «l'acte illocutionnaire est (...) l'acte qui, en plus de tout ce qu'il fait en tant qu'il est aussi une locution (i.e. en tant qu'il dit quelque chose), produit quelque chose en disant ${ }^{1} »$. Nous nous sommes par ailleurs largement inspirés de la typologie établie à ce propos par Récanati. Rappelons que «Récanati a proposé une classification arborescente dont la cohérence se fonde essentiellement sur le principe d'ajustement. La raison de ce choix est la suivante : ce critère permet de classer en même temps et les états psychologiques et les buts illocutionnaires ${ }^{2}$. » C'est sur cette base que cet auteur distingue parmi les actes illocutionnaires, les actes essentiellement représentatifs des actes non essentiellement représentatifs. Les premiers se définissent à partir du contenu propositionnel qu'ils véhiculent et les seconds par leur caractère expressif. Les actes essentiellement représentatifs se subdivisent à leur tour en deux catégories : les actes performatifs qui laissent les mots exercer leur pouvoir sur le monde, et les actes constatifs qui à l'inverse procèdent d'une captation du monde par le biais des mots. Selon Récanati, l'action des actes performatifs s'exerce de trois manières différentes -la déclaration, la promesse et la prescriptiondonnant lieu aux trois actes correspondants que sont les déclaratifs, les promissifs et les prescriptifs.

\footnotetext{
J-P. MEUNIER, D. PERAYA, op.cit., p. 82.

2 J-P. MEUNIER, D. PERAYA, op.cit., p. 92.
} 


\section{La presse spécialisée}

Dans les articles de la presse spécialisée, on constate globalement un recours privilégié aux actes constatifs qui constituent véritablement la grande majorité des actes du discours. Si à l'occasion l'un ou l'autre prescriptif apparait, il ne constitue jamais une injonction au lecteur, mais il livre plutôt l'avis du journaliste comme en attestent les extraits suivants :

«Force est de constater que les fonds de pension qui ont réalisé les meilleurs scores sont cependant ceux qui se sont le plus délestés de leurs actions en cours d'année au bénéfice d'obligations surtout américaines. »

(L'Echo, «Une année de perdue pour les fonds de pension : le return moyen s'élève à $-0,1 \%$ en 2000 », 29/03/2001)

« Les distributeurs européens devront également faire face à des challenges opérationnels, susceptibles d'engendrer des coûts supplémentaires...»

(L'Echo, «Belle croissance bénéficiaire en perspective pour les distributeurs alimentaires européens », 07/06/2001)

\section{La presse non spécialisée}

Au niveau de la presse non spécialisée, on retrouve une distinction entre Le Vif/L'Express et La Libre Belgique. Les actes relevés dans les articles de La Libre Belgique restent globalement de l'ordre du constatif alors que ceux répertoriés dans Le Vif se caractérisent par la récurrence de prescriptifs constituant parfois de véritables injonctions à l'adresse du lecteur ${ }^{1}$. L'un des cas les plus exemplatifs nous est fourni par l'article «Intéressants les cliquets ?» (25/08/2000). L'ensemble de cet article s'articule autour de quelques grands « tuyaux » que le journaliste confie au lecteur à propos des sicavs à cliquets :

«Tuyau ${ }^{\circ} 1$ : la lecture attentive du prospectus d'émission vous permettra de savoir avec précision quelles sont les modalités de remboursement de vos parts à l'échéance. »

1 On comptabilise une moyenne de 5 à 6 prescriptifs par article. 


\section{Hétérogénéité des profils relationnels}

Au départ de ces quatre niveaux d'analyse pragmatique, nous avons tenté de caractériser le type de relation qui s'établit entre le journaliste et le lecteur dans les divers journaux et magazines passés en revue. Il nous semble à présent que nous sommes en mesure de proposer pour chacun d'entre eux un profil relationnel spécifique susceptible de nous éclairer sur la transmission des savoirs économiques et boursiers informels.

\section{Trends Tendances : une information spécialisée à portée réflexive dans le cadre d'une relation informative d'expert à expert}

Nos analyses ont démontré que les articles du Trends Tendances se caractérisent globalement par une faible implication pronominale, peu de marques journalistiques, un discours essentiellement constatif et l'exploitation d'une logique authentifiante notamment assurée par le recours à des intervenants prestigieux, par certaines marques journalistiques, ainsi que par un mode de signature spécifique ${ }^{1}$.

Ces divers constats plaident en faveur d'un journaliste avant tout soucieux de faire le point sur une question boursière d'ordre général (par exemple au sujet des revendications des actionnaires externes concernant les structures complexes d'actionnariat mises en place par les entreprises; le changement des Bourses qui tendent à se présenter comme des entreprises; etc.), s'impliquant peu ou du moins très indirectement dans son propos et n'interpellant pas de façon immédiate son destinataire. Tous ces éléments nous incitent à qualifier le journaliste en tant que témoin averti ou témoin expert établissant une relation de l'ordre de la distanciation avec son lectorat, lui aussi posé comme étant averti. Le modèle relationnel établi, la présentation relativement complète, nuancée et distante de l'information boursière ainsi que le degré de savoirs boursiers supposés partagés par le lecteur, semblent favoriser l'engagement de ce dernier dans une démarche réflexive et inférentielle.

\footnotetext{
1 Les articles étudiés sont signés « The Economist ».
} 


\section{L'Echo : une information spécialisée dans le cadre d'une relation indirectement conseillère d'expert à expert}

Nous avons vu que les articles de L'Echo se caractérisent eux aussi par une implication pronominale globalement faible ainsi que par un net privilège aux marques journalistiques authentifiantes. Cette logique de légitimation du discours journalistique semble prendre ici une place tout à fait centrale : elle se traduit entre autres par un recours systématique aux intervenants prestigieux dont les propos sont suivis de très près par le journaliste ${ }^{1}$ ainsi que par une présence abondante de références ${ }^{2}$. Le journaliste demeure donc relativement effacé derrière les propos des intervenants et cela à tous les niveaux du discours envisagés. En conservant par ailleurs un ton exclusivement constatif, le journaliste apparaît comme un témoin, un intermédiaire chargé de relater des propos d'experts à des lecteurs avertis et clairement définis en filigrane comme des investisseurs. Sans véritablement entrer dans une logique conseillère, le journaliste relaie une série d'informations pointues susceptibles d'orienter les comportements d'investissement de ses lecteurs, encouragés à s'engager dans une démarche inférentielle.

La Libre Entreprise : une information spécialisée dans le cadre d'une relation conseillère entre un « journaliste-relais » averti et engagé et un « lecteur-investisseur » averti.

Les articles issus de La Libre Entreprise se caractérisent quant à eux par une implication pronominale atténuée mais bien présente (multiplication des « on »), par des marques journalistiques plutôt impliquantes (récurrence des questions) ainsi que par une logique authentifiante relative (recours aux intervenants prestigieux et nombreuses références). Autrement dit, alors même que le journaliste se positionne de façon assez ouverte, il n'en prend pas moins le soin de légitimer ses propos. Le journaliste ne se présente donc pas comme un simple témoin mais bien comme un médiateur engagé. Selon les articles, il apparaît comme un investigateur rapportant son point de vue sur des faits ou comme établissant un bilan hebdomadaire sur les valeurs boursières. On se

1 Rappelons à ce propos que chaque article se contente de présenter les résultats d'une enquête ou d'une étude réalisée par une institution reconnue telle une banque par exemple.

2 On note également une auto-référence. 
rapproche d'une certaine façon de la logique conseillère de L'Echo à la différence près que le conseil se gonfle d'une plus grande distance et qu'il est surtout le fait d'un journaliste engagé.

Tout cela nous incite à caractériser la relation entre le journaliste et le lecteur comme une relation conseillère mettant en présence un journaliste averti et engagé et un lecteur défini comme un investisseur averti.

La Libre Belgique : une information thématique dans le cadre d'une relation implicitement conseillère entre un «journaliste-relais » effacé et un « lecteur-investisseur» averti.

Les articles des pages boursières courantes de La Libre Belgique présentent un effacement particulièrement fort des marques pronominales et des marques journalistiques y compris au niveau des actes du discours qui demeurent purement constatifs et au niveau de la signature toujours réduite à des initiales. La logique d'authentification des propos reste quant à elle présente bien qu'atténuée : si la plupart des intervenants conservent un certain prestige, ils réfèrent néanmoins davantage à des catégories générales (analystes) ou à des institutions (Fortis, KBC Securities, etc.) qu'à des individus précisément définis. Le journaliste reste particulièrement effacé et se présente de ce fait comme un témoin objectif se contentant de relater une série d'informations (des évaluations d'experts) susceptibles d'intéresser un lectorat d'investisseurs avertis. Cela nous incite à parler d'une forme de relation conseillère implicite dans la mesure où le conseil n'est pas donné comme tel mais peut résulter d'une démarche inférentielle propre au lecteur.

Le Vif/L'Express : une information semi vulgarisée dans le cadre d'une relation explicitement conseillère entre un journaliste non expert engagé et un « lecteur-investisseur» néophyte.

Les articles du Vif/L'Express se distinguent quant à eux par une implication pronominale marquée et directe (recours fréquent au pronom « vous »), par le privilège accordé aux marques journalistiques fortement impliquantes (multiplication des questions), par une présence relative d'actes prescriptifs et corrélativement par une absence presque totale de marques authentifiantes (les intervenants apparaissent à titre exceptionnel, restent vagues et ancrent des « adages populaires »; les références sont également absentes). On se trouve ici face à un journa- 
liste nettement engagé dans une logique conseillère de proximité auprès d'un lecteur positionné comme un investisseur « débutant».

\section{Conclusion}

Au terme de ce ratissage pragmatique de la presse boursière, il apparaît que la relation qui s'instaure entre le journaliste et le lecteur dépend prioritairement du type d'information véhiculé par le journal et plus globalement encore, de la ligne éditoriale suivie par ce dernier. De ce point de vue, la distinction établie entre la presse dite spécialisée et la presse non spécialisée s'avère éclairante. De façon assez schématique, nous pouvons considérer que deux dimensions caractérisent le positionnement de chacun des interlocuteurs. Selon les cas, le journaliste se présente comme étant plus ou moins expert ou non dans la thématique boursière et comme étant plus ou moins engagé ou non dans son propos. De même, le lecteur se trouve défini par des degrés d'expertise variables et désigné de façon plus ou moins implicite ou explicite par le discours journalistique. Nous l'avons vu, le croisement de ces dimensions débouche sur plusieurs profils relationnels. L'intérêt de ces profils relationnels réside surtout dans ce qu'ils nous révèlent -au départ de l'option pragmatique développée dans chaque article- sur le type de savoirs économiques et boursiers transmis, mais aussi sur les opportunités d'apprentissages informels.

Les profils construits autour d'interlocuteurs experts -que l'on trouve notamment dans le Trends Tendances, L'Echo et La Libre Entreprise- mettent en scène des savoirs boursiers pointus qui supposent l'acquisition préalable d'un certain niveau de connaissances et donc le partage effectif de la base de connaissances présupposée pour la compréhension de l' article, cela d' autant plus si le lecteur souhaite saisir les enjeux sous-jacents auxquels ces articles spécialisés renvoient ${ }^{1}$. On

1 Nous visons ici tout le versant «implicite» du discours boursier de la presse, autrement dit l'ensemble des inférences et des raisonnements sur lesquels les informations transmises peuvent déboucher. À noter à ce propos le statut tout à fait particulier qui caractérise l'information boursière en termes d'impact réel et cela en raison du jeu d'anticipation qui guide largement les comportements des investisseurs et dès lors aussi l'évolution du marché boursier en tant que tel. En effet, la simple divulgation d'une information -ou même l'anticipation de cette divulgation et de son impact potentiel- exerce un effet sur le comportement réel d'achat et de vente des investisseurs, influençant directement le cours des actions et donc le marché luimême. 
peut supposer que les lecteurs avertis auxquels s'adressent prioritairement ces articles trouvent de fait un ensemble d'informations significatives qui leurs permettent d'étoffer un certain nombre de leurs connaissances. Les savoirs ainsi intégrés n'en demeurent pas moins lacunaires dans la mesure où ils relèvent davantage du complément d'information, du point de détail ou de l'information générique ${ }^{1}$. Dans cette perspective, la presse boursière spécialisée apparaît surtout comme le support d'un complément informatif spécialisé (adressé à un lectorat averti), un moyen de maintenir ses connaissances et ses informations à jour ou de les recycler, ainsi que comme un pourvoyeur de connaissances génériques, incomplètes et peu structurées présupposant l'accès à d'autres sources d'informations.

S'agissant des profils engageant des interlocuteurs peu avertis, voire néophytes comme dans le cadre du Vif/L'Express par exemple, ils se distinguent sans véritable surprise tant dans leur stratégie relationnelle que dans le type de savoirs qu'ils convoquent et véhiculent. Les savoirs transmis restent spécialisés pour une part, mais ils s'intègrent également dans un discours plus généraliste, plus «vulgarisé » -sans l'être pour autant réellement- et donc plus accessible du moins à première vue. En effet, bien que présentés comme non experts, les lecteurs doivent pour comprendre ces articles, disposer d'un certain nombre de connaissances préalables sur la bourse. Or il y a fort à parier -notamment au vu du type de relation conseillère que l'on trouve dans certains articles du Vif/L'Express ${ }^{2}$ - que la plupart des lecteurs auxquels s'adressent ces articles ne possèdent ni les savoirs présupposés, ni les connaissances suffisantes pour cerner les enjeux réels du système boursier alors même qu'ils sont invités -entre autres par les appels confiants que leurs lancent certaines institutions- à nourrir la croyance inverse. Globalement, les articles du Vif/L'Express et de La Libre Belgique véhiculent donc aussi des savoirs économiques et boursiers génériques, lacunaires et peu structurés mais cette fois non plus à titre de complément informatif mais bien en tant que base de connaissances. Dans la mesure où les lecteurs concernés ne disposent vraisemblablement pas des assises conceptuelles requises pour intégrer les savoirs transmis,

1 Nous faisons ici allusion à la structuration des connaissances par niveaux telle que l'envisage E. ROSCH dans le processus de catégorisation mentale. Voir à ce propos E. ROSCH, B. B. LLOYD, Cognition and categorisation, Hillsdale (NJ), Erlbaum, Lawrence, Associates, 1978.

2 Cet aspect a été plus longuement développé dans un article ultérieur. 
ce type de discours médiatique ne peut offrir aux moins avertis qu'une connaissance et une compréhension superficielle, incomplète et peu structurée et cela bien que le discours de cette presse tende à induire une forme de proximité et l'impression d'une plus grande accessibilité du système boursier.

En finale, il apparaît qu'indépendamment du degré de spécialisation de la presse boursière, le rôle des connaissances préalables reste essentiel pour que le lecteur puisse prétendre à l'intégration effective et efficace des savoirs transmis. Un complément informatif doit donc être recherché ailleurs par le lecteur, et cela tant en aval qu'en amont de ses lectures de la presse. Cela implique la mise en ouvre d'un processus cognitif de comparaison ${ }^{1}$ et de recoupement des informations recueillies à partir de sources diverses, ce processus opérant également au sein même de chaque article. Cela nous incite à postuler que les apprentissages s'effectuent globalement sur le mode du contact, de la répétition, menant du point de vue des non initiés, davantage à un mode de familiarisation avec le jargon boursier et ses mécanismes qu'à sa compréhension réelle. Par ailleurs, le lecteur peu averti expérimente très probablement cette familiarisation par le biais des représentations schématiques et manichéennes ${ }^{2}$ qu'il se construit au fil de ses lectures répétées.

Ces quelques pistes de réflexion trouveront un écho et un enrichissement au terme des différents travaux présentés à partir de l'analyse du corpus, chaque ratissage entretenant des interactions étroites avec les autres. Par ailleurs, si ces premières hypothèses méritent d'être approfondies, il importe également d'insister sur l'intérêt d'une étude plus empirique seule à même de révéler les connaissances boursières effectivement acquises par la lecture de la presse ainsi que le niveau de connaissance réellement partagé par le lectorat.

1 Nous faisons ici allusion au processus comparatif tel que le définit R. LANGACKER, Foundations of cognitive grammar, Theoretical prerequisites, vol.I., Stanford (Ca), Stanford University Press, 1987.

2 Le ratissage métaphorique s'avère particulièrement éclairant à ce propos, envisagé au départ des travaux de G. LAKOFF, M. JOHNSON, Les métaphores dans la vie quotidienne, Paris, Editions de Minuit, 1985. Par le qualificatif «schématiques» nous visons directement la notion de schéma telle que la définissent NORMAN et RUMELHART soit des blocs de connaissances de niveaux divers susceptibles de s'appeler mutuellement.. Voir à ce sujet D. RUMELHART, D. NORMAN, «Les schémas », in La psychologie, Textes Essentiels, Paris, Larousse, 1983. 
